\title{
A time-dependent first-order Bethe-Goldstone theory $\dagger$
}

\author{
Gy Csanakł and V McKoy \\ \$Instituto de Fisica, Universidade Estadual de Campinas, CP 1170, 13100 Campinas, \\ Sao Paulo, Brazil \\ $\$$ Arthur Amos Noyes Laboratory of Chemical Physics $\mid$, California Institute of Tech- \\ nology, Pasadena, California 91125, USA
}

Received 31 May 1977, in final form 15 August 1977

\begin{abstract}
The time-dependent first-order Bethe-Goldstone theory is defined by using the Hartree-Fock approximation in the Bethe-Goldstone equation with the external field fully coupled in. Starting from this equation and using the Gell-Mann and Low projection technique various amplitude equations are obtained. Finally an equation is obtained in differential form for the excited-state pair amplitude. This can be used to calculate transition moments between the ground and excited states of atomic or molecular systems which incorporate short-range correlation effects.
\end{abstract}

\section{Introduction}

The total non-relativistic wavefunction of an atomic or molecular system can be considered as consisting of two parts: the Hartree-Fock (HF) approximation and the correlation part (Löwdin 1959, Sinanoğlu and Brueckner 1970, Schaefer 1972). Effects connected with the correlation part of the wavefunction are called correlation effects. The correlation energy is defined as the difference between the exact nonrelativistic energy and the $\mathrm{HF}$ energy.

Early attempts at calculating the correlation energy and other correlation effects have been summarised by Löwdin (1959) and a bibliography given by Yoshizumi (1959). The most general early approach has been the configuration-interaction (CI) method (Löwdin 1959, Parr 1963, Schaefer 1972). This method can be slowly convergent and the number of configurations increases rapidly with the number of electrons in the system. Moreover, the method may not always provide much physical insight about the nature of electron correlation. A more physical approach to the correlation problem has been taken by Sinanoğlu through the introduction of pair-correlation theories. Sinanoğlu and co-workers (Sinanoğlu 1961, 1962a, b, Sinanoğlu and Tuan 1963, Tuan and Sinanoğlu 1964, McKoy and Sinanoğlu 1964) have analysed the correlation problem and have shown that the many-electron correlation problem can be reduced to a set of two-electron problems and that the total wavefunction can be well approximated by the one- and two-electron parts. Further support for the pair-correlation theory came from the application of many-body perturbation theory (MBPT) to the atomic correlation problem by Kelly $(1963,1964)$. It has been shown (Freed 1968) that the most important diagrams that contribute to the

$\dagger$ Work supported by the NSF(OIP)-CNPq (Brasil) Cooperative Science Program.

Contribution No 5583. 
correlation energy are those that are inherently included in Sinanoǧlu's pair theory and they are of a short-range nature.

Sinanoğlu also related his pair theory to one so-called Bethe-Goldstone pair theory (Bethe and Goldstone 1957) used in the theory of nuclear matter. He pointed out that for most atomic and molecular systems the HF approximation is a good starting point and that there is no need for a self-consistency requirement between the one- and two-particle clusters, which was essential for nuclear matter. Hence the two theories can be expected to give essentially identical results for atoms and molecules. Nesbet (1969) has also applied the Bethe-Goldstone theory extensively to problems of correlation in atoms and molecules. The Bethe-Goldstone theory can be formulated by the many-body Green's function technique of Martin and Schwinger (1959) and consequently the theory of short-range correlations can be put into the context of this formalism. The Bethe-Goldstone equation with the HF one-particle Green's function will be called the first-order Bethe-Goldstone equation (Meneses and Csanak 1975).

On the other hand, in the calculation of frequency-dependent properties or transition moments between the ground and excited states, the most widely used scheme has been the time-dependent Hartree-Fock (TDHF) or random-phase approximation (RPA) (for reviews on the RPA see, for example, Lane 1964, McLachlan and Ball 1964, Dalgarno 1966, Rowe 1968). In this scheme the HF approximation is assumed for the atomic or molecular electrons in the presence of the external field. We refer here to the fully coupled time-dependent HF method. The TDHF or RPA method can also be formulated in the context of the many-body Green's function technique (Baym and Kadanoff 1961).

The TDHF method was first used by Dalgarno and Victor (1966) in the calculation of atomic polarisabilities and by Altick and Glassgold (1964) and Dunning and McKoy (1967) in the calculation of transition moments for atoms and molecules respectively. Since then a large number of calculations have been done for atomic and molecular systems using the TDHF method (for recent reviews see, for example, McCurdy et al 1977, Wendin 1976, Amusia and Cherepkov 1975).

A natural extension of the TDHF method would be to introduce correlation effects between the electrons while the external field is coupled in. As discussed earlier, the most important correlation effect in the field-free case proved to be the direct short-range correlations which are generally referred to as 'pair correlations'. It can be assumed that the same correlation effect plays the central role in the case of a weak external field which is important for linear response properties and transition moments.

In this paper we develop a theory using the many-body Green's function technique which introduces short-range correlation effects in the presence of an external field. This theory can be called the time-dependent first-order Bethe-Goldstone theory. The external field will be considered exactly, i.e. it will be 'fully coupled in'. In $\$ 2$ the basic equations of the theory will be discussed, whilst $\$ 3$ will transform the final equation of $\$ 2$ to energy space and the final equation of the whole theory and its potential applications will be discussed.

\section{Basic equations of the time-dependent first-order Bethe-Goldstone theory}

In this theory the first-order Bethe-Goldstone equation will be used in the presence 
of an arbitrary time-dependent external potential $U(1) \equiv U\left(\boldsymbol{r}_{1}, \sigma_{1}, t_{1}\right)(\boldsymbol{r}, \sigma$ and $t$ are the spatial, spin and time variables respectively) where, as discussed above, first-order refers to the fact that the one-particle Green's function will be used in the HF approximation (for the definition of the multi-particle Green's function and the general notation used here see Csanak et al (1971)). Thus we have

$$
\begin{aligned}
G_{2}^{\mathrm{BG} 1}\left(1,2,1^{\prime} 2^{\prime} ; U\right) \\
=G_{\mathrm{HF}}\left(1,1^{\prime} ; U\right) G_{\mathrm{HF}}\left(2,2^{\prime} ; U\right)-G_{\mathrm{HF}}\left(1,2^{\prime} ; U\right) G_{\mathrm{HF}}\left(2,1^{\prime} ; U\right) \\
\quad+\mathrm{i} \int \mathrm{d} \overline{1} \mathrm{~d} \overline{2} G_{\mathrm{HF}}(1, \overline{1} ; U) G_{\mathrm{HF}}(2, \overline{2} ; U) V(\overline{1}-\overline{2}) G_{2}^{\mathrm{BG} 1}\left(\overline{1} \overline{2}, 1^{\prime} 2^{\prime} ; U\right)
\end{aligned}
$$

where $G_{\mathrm{HF}}\left(1,1^{\prime} ; U\right)$ is the one-particle Green's function in the HF approximation with the external field coupled in (TDHF theory), and $G_{2}^{\mathrm{BG} 1}\left(12,1^{\prime} 2^{\prime} ; U\right.$ ) is the two-particle Green's function in the first-order Bethe-Goldstone (BG1) approximation with the external field coupled in, i.e. equation (1) defines our approximation scheme. This equation is formally identical to equation (53) of Baym and Kadanoff (1961) except that $G\left(1,1^{\prime} ; U\right)$ is replaced by $G_{\mathrm{HF}}\left(1,1^{\prime} ; U\right)$. Also this equation with $U=0$ defines the first-order Bethe-Goldstone theory. In equation (1) the integrations denote an integration over the spatial and time coordinates and a summation over the spin variables, and $V(1-2)=V\left(r_{1}-r_{2}\right) \delta\left(t_{1}-t_{2}\right)$ where $V\left(r_{1}-r_{2}\right)$ is the Coulomb potential.

In order to calculate the response of the correlation effect to the external field, the functional derivative of equation (1) will be taken and the Schwinger equation used in the form (Martin and Schwinger (1959); see also Kato et al (1960) for a detailed discussion):

$$
\left.\frac{\delta G_{2}\left(12,1^{\prime} 2^{\prime} ; U\right)}{\delta U(3)}\right|_{U=0}=-G_{3}\left(123,1^{\prime} 2^{\prime} 3^{+}\right)+G_{2}\left(12,1^{\prime} 2^{\prime}\right) G\left(3,3^{+}\right)
$$

where $G_{3}\left(123,1^{\prime} 2^{\prime} 3^{\prime}\right)$ is the three-particle Green's function. After taking the functional derivative of equation (1) the Gell-Mann and Low (1951) projection operator can be used to obtain an equation for the two-particle transition amplitude

$$
\rho_{0 n}\left(12,1^{\prime} 2^{\prime}\right)=\left\langle 0^{N}\left|T\left(\psi(1) \psi(2) \psi^{\dagger}\left(2^{\prime}\right) \psi^{\dagger}\left(1^{\prime}\right)\right)\right| n^{N}\right\rangle
$$

where $\left|0^{N}\right\rangle$ and $\left|n^{N}\right\rangle$ are the ground and excited states respectively of an $N$-electron system and $\psi(1)$ is the field operator in the Heisenberg representation. We obtain an expression for this transition amplitude of the form

$$
\begin{aligned}
\rho_{0 n}^{\mathrm{BG} 1}\left(12,1^{\prime} 2^{\prime}\right)= & \mathrm{i} X_{0}^{n, \mathrm{RPA}}\left(1,1^{\prime}\right) G_{\mathrm{HF}}\left(2,2^{\prime}\right)+\mathrm{i} G_{\mathrm{HF}}\left(1,1^{\prime}\right) X_{0}^{n, \mathrm{RPA}}\left(2,2^{\prime}\right) \\
& -\mathrm{i} X_{0}^{n, \mathrm{RPA}}\left(1,2^{\prime}\right) G_{\mathrm{HF}}\left(2,1^{\prime}\right)-\mathrm{i} G_{\mathrm{HF}}\left(1,2^{\prime}\right) X_{0}^{n, \mathrm{RPA}}\left(2,1^{\prime}\right) \\
& -\int \mathrm{d} \overline{1} \mathrm{~d} \overline{2} X_{0}^{n, \mathrm{RPA}}(1, \overline{1}) G_{\mathrm{HF}}(2, \overline{2}) V(\overline{1}-\overline{2}) G_{2}^{\mathrm{BG} 1}\left(\overline{1} \overline{2}, 1^{\prime} 2^{\prime}\right) \\
& -\int \mathrm{d} \overline{1} \mathrm{~d} \overline{2} G_{\mathrm{HF}}(1, \overline{1}) X_{0}^{n, \mathrm{RPA}}(2, \overline{2}) V(\overline{1}-\overline{2}) G_{2}^{\mathrm{BG} 1}\left(\overline{1} \overline{2}, 1^{\prime} 2^{\prime}\right) \\
& +\mathrm{i} \int \mathrm{d} \overline{1} \mathrm{~d} \overline{2} G_{\mathrm{HF}}(1, \overline{1}) G_{\mathrm{HF}}(2, \overline{2}) V(\overline{1}-\overline{2}) \rho_{0 n}^{\mathrm{BG} 1}\left(\overline{1} \overline{2}, 1^{\prime} 2^{\prime}\right)
\end{aligned}
$$

where $X_{0}^{n, \mathrm{RPA}}\left(1,1^{\prime}\right)$ is the RPA to the transition amplitude defined by

$$
X_{0}^{n}\left(1,1^{\prime}\right)=\left\langle 0^{N}\left|T\left(\psi(1) \psi^{\dagger}\left(1^{\prime}\right)\right)\right| n^{N}\right\rangle
$$


(for a detailed discussion of $X_{0}^{n, R P A}\left(1,1^{\prime}\right)$ see Csanak (1974)). The Gell-Mann and Low operation can be applied once more to equation (4) to yield an equation for the amplitude $\chi_{l}^{n, \mathrm{BG} 1}(1,2)$, which is the first-order Bethe-Goldstone approximation to the amplitude defined by

$$
\chi_{l}^{n}(1,2)=\left\langle l^{N-2}|T(\psi(1) \psi(2))| n^{N}\right\rangle
$$

where $\left|l^{N-2}\right\rangle$ is an arbitrary state of the $(N-2)$-electron system. $\chi_{l}^{n}(1,2)$ can be considered as the pair amplitude of the excited state $\left|n^{N}\right\rangle$. The equation so obtained has the following form

$$
\begin{aligned}
\chi_{l}^{n, \mathrm{BG} 1}(12)= & \int \mathrm{d} \overline{1} \mathrm{~d} \overline{2} X_{0}^{n, \mathrm{RPA}}(1, \overline{1}) G_{\mathrm{HF}}(2, \overline{2}) V(\overline{1}-\overline{2}) \chi_{l}^{\mathrm{BG} 1}(\overline{1} \overline{2}) \\
& +\int \mathrm{d} \overline{1} \mathrm{~d} \overline{2} G_{\mathrm{HF}}(1, \overline{1}) X_{0}^{n, \mathrm{RPA}}(2, \overline{2}) V(\overline{1}-\overline{2}) \chi_{l}^{\mathrm{BGi} 1}(\overline{1} \overline{2}) \\
& +\mathrm{i} \int \mathrm{d} \overline{1} \mathrm{~d} \overline{2} G_{\mathrm{HF}}(1, \overline{1}) G_{\mathrm{HF}}(2, \overline{2}) V(\overline{1}-\overline{2}) \chi_{l}^{n, \mathrm{BG} 1}(\overline{1} \overline{2})
\end{aligned}
$$

where $\chi_{l}^{\mathrm{BG} 1}(12)$ is the first-order ground-state Bethe-Goldstone amplitude which satisfies the equation

$$
\chi_{l}^{\mathrm{BG} 1}(12)=\mathrm{i} \int G_{\mathrm{HF}}(1, \overline{1}) G_{\mathrm{HF}}(2, \overline{2}) V(\overline{1}-\overline{2}) \chi_{l}^{\mathrm{BG} 1}(\overline{1} \overline{2}) \mathrm{d} \overline{1} \mathrm{~d} \overline{2}
$$

and it is the first-order Bethe-Goldstone approximation to the amplitude

$$
\chi_{i}(1,2)=\left\langle l^{N-2}|T(\psi(1) \psi(2))| 0^{N}\right\rangle .
$$

Equation (7) is the fundamental equation of the first-order time-dependent BetheGoldstone theory.

\section{Analytical details}

Equation (7) will now be put into an analytical form which is more convenient for numerical analysis. To do this we will transform equation (7) so as to replace the time variable by the energy variable, i.e. by Fourier transformation. First we note that on the right-hand side of equation (7) both the amplitudes $\chi_{l}(\overline{1}, \overline{2})$ and $\chi_{l}^{n}(\overline{1}, \overline{2})$ appear in the time-contracted form because they are multiplied by $V(\overline{1}-\overline{2})$ which contains a factor of $\delta\left(t_{\overline{1}}-t_{\overline{2}}\right)$. Hence equation (7) can be 'closed' for the time-contracted amplitude and on the left-hand side we can also choose $t_{2}=t_{1}^{+}$ $\left(t_{1}^{+} \equiv t_{1}+\epsilon\right.$ where $\left.\epsilon \rightarrow 0^{+}\right)$. In the time-contracted amplitudes the following factorisations can be used

$$
\begin{aligned}
& \chi_{l}\left(12^{+}\right)=\exp \left(-i \tilde{\epsilon}_{n} t_{1}\right) \chi_{l}\left(r_{1} r_{2}\right) \\
& \chi_{l}^{n}\left(12^{+}\right)=\exp \left[-i\left(\tilde{\epsilon}_{l}+\omega_{n}\right) t_{1}\right] \chi_{l}^{n}\left(r_{1} r_{2}\right) \\
& X_{0}^{n}\left(1,1^{\prime}\right)=\exp \left[-i \omega_{n}\left(t_{1}+t_{2}\right) / 2\right] X_{0}^{n}\left(r_{1} r_{1}^{\prime} ; t_{1}-t_{1}^{\prime}\right)
\end{aligned}
$$

(for (10a) see Meneses and Csanak (1975), for (10c) see Csanak (1974), and (10b) can be proven analogously) where

$$
\tilde{\epsilon}_{l}=E_{0}^{N}-E_{l}^{N-2}
$$


is the pair energy and

$$
\omega_{n}=E_{n}^{N}-E_{0}^{N}
$$

is the excitation energy of the $N$-electron system and

$$
\begin{aligned}
& \chi_{l}\left(r_{1} r_{2}\right)=\left\langle l^{N-2}\left|\psi\left(r_{1}\right) \psi\left(r_{2}\right)\right| 0^{N}\right\rangle \\
& \chi_{l}^{n}\left(r_{1} r_{2}\right)=\left\langle l^{N-2}\left|\psi\left(r_{1}\right) \psi\left(r_{2}\right)\right| n^{N}\right\rangle .
\end{aligned}
$$

Also $E_{0}^{N}$ is the energy of the ground state $\left|0^{N}\right\rangle$ and $E_{l}^{N-2}$ is that of the state $\left|l^{N-2}\right\rangle$. Using the factorisations of $(10 a, b, c)$ and Fourier transforming we obtain from equation (7)

$$
\begin{aligned}
\chi_{l}^{n, \mathrm{BG} 1}\left(r_{1} r_{2}\right)= & \frac{1}{2 \pi} \int \mathrm{d} z \int \mathrm{d} \bar{r}_{1} \mathrm{~d} \bar{r}_{2} X_{0}^{n, \mathrm{RPA}}\left(r_{1} \bar{r}_{1} ; z\right) G_{\mathrm{HF}}\left(r_{2} \bar{r}_{2} ; \frac{1}{2} \omega_{n}+\tilde{\epsilon}_{l}-z\right) \\
& \times V\left(\bar{r}_{1}-\bar{r}_{2}\right) \chi_{l}^{\mathrm{BG} 1}\left(\bar{r}_{1} \bar{r}_{2}\right)+\frac{1}{2 \pi} \int \mathrm{d} z \int \mathrm{d} \bar{r}_{1} \mathrm{~d} \bar{r}_{2} G_{\mathrm{HF}}\left(r_{1} \bar{r}_{1} ; z\right) \\
& \times X_{0}^{n, \mathrm{RPA}}\left(r_{2} \bar{r}_{2} ; \frac{1}{2} \omega_{n}+\tilde{\epsilon}_{l}-z\right) V\left(\bar{r}_{1}-\bar{r}_{2}\right) \chi_{l}^{\mathrm{BG} 1}\left(\bar{r}_{1} \bar{r}_{2}\right)+\frac{1}{2 \pi} \int \mathrm{d} z \int \mathrm{d} \bar{r}_{1} \mathrm{~d} \bar{r}_{2} \\
& \times G_{\mathrm{HF}}\left(r_{1} \bar{r}_{1} ; z\right) G_{\mathrm{HF}}\left(r_{2} \bar{r}_{2} ; \frac{1}{2} \omega_{n}+\tilde{\epsilon}_{l}-z\right) V\left(\bar{r}_{1}-\bar{r}_{2}\right) \chi_{l}^{n, \mathrm{BG} 1}\left(\bar{r}_{1} \bar{r}_{2}\right)
\end{aligned}
$$

where $X_{0}^{n, \mathrm{RPA}}\left(r_{1} \bar{r}_{1} ; z\right)$ and $G_{\mathrm{HF}}\left(r_{1} \bar{r}_{1} ; z\right)$ are the Fourier transforms of $X_{0}^{n, \mathrm{RPA}}\left(r_{1} \bar{r}_{1} ; t_{1}-t_{\overline{1}}\right)$ and $G_{\mathrm{HF}}\left(r_{1} r_{1} ; t_{1}-t_{\overline{1}}\right)$ respectively. These analytical definitions are discussed in Csanak et al (1971) and Csanak (1974).

We now simplify equation (15) and put it in differential form. For the ground-state pair-correlation function this was achieved by equation (9) of Meneses and Csanak (1975). This can be achieved through the calculation of the convolution integral on the right-hand side of equation (15) using the spectral representations of $G_{\mathrm{HF}}(r \bar{r} ; z)$ and $X_{0}^{n, R P A}(r \bar{r} ; z)$ (Csanak 1974). After considerable manipulation we obtain the following relatively simple form

$$
\begin{aligned}
{\left[\left(\tilde{\epsilon}_{l}+\omega_{n}\right)-\right.} & \left.h_{\mathrm{HF}}\left(r_{1}\right)-h_{\mathrm{HF}}\left(r_{2}\right)\right] \chi_{l}^{n}\left(r_{1} r_{2}\right) \\
= & V\left(r_{1}-r_{2}\right) \chi_{l}^{n}\left(r_{1} r_{2}\right)-\int \rho^{\mathrm{HF}}\left(r_{1} \bar{r}_{1}\right) V\left(\bar{r}_{1}-r_{2}\right) \chi_{l}^{n}\left(\bar{r}_{1} r_{2}\right) \mathrm{d} \bar{r}_{1} \\
& -\int \rho^{\mathrm{HF}}\left(r_{2} \bar{r}_{2}\right) V\left(r_{1}-\bar{r}_{2}\right) \chi_{l}^{n}\left(r_{1} \bar{r}_{2}\right) \mathrm{d} \bar{r}_{2} \\
& -\int X_{n}^{\mathrm{RPA}}\left(r_{1} \bar{r}_{1}\right) V\left(r_{2}-\bar{r}_{1}\right) \chi_{l}\left(\bar{r}_{1} r_{2}\right) \mathrm{d} \bar{r}_{1}+\int V_{0 n}^{\mathrm{RPA}}\left(r_{1}^{\prime} r_{1}\right) \chi_{l}\left(r_{1}^{\prime} r_{2}\right) \mathrm{d} r_{1}^{\prime} \\
& -\int X_{n}^{\mathrm{RPA}}\left(r_{2} \bar{r}_{2}\right) V\left(r_{1}-\bar{r}_{2}\right) \chi_{l}\left(r_{1} \bar{r}_{2}\right) \mathrm{d} \bar{r}_{2}+\int V_{0 n}^{\mathrm{RPA}}\left(r_{2}^{\prime} r_{2}\right) \chi_{l}\left(r_{1} r_{2}^{\prime}\right) \mathrm{d} r_{2}^{\prime}
\end{aligned}
$$

where $h_{\mathrm{HF}}(r)$ is the Hartree-Fock operator defined by

$$
h_{\mathrm{HF}}(r) \phi(r)=\left(-\frac{1}{2} \nabla^{2}+\frac{Z}{r}\right) \phi(r)+\int \Sigma_{\mathrm{HF}}\left(r_{1} r^{\prime}\right) \phi\left(r^{\prime}\right) \mathrm{d} r^{\prime}
$$

and $\Sigma_{\mathrm{HF}}\left(r r^{\prime}\right)$ is the $\mathrm{HF}$ potential

$$
\Sigma_{\mathrm{HF}}\left(r r^{\prime}\right)=\delta\left(r-r^{\prime}\right) \int V\left(r-r_{2}\right) \rho^{\mathrm{HF}}\left(r_{2} r_{2}\right) \mathrm{d} r_{2}-V\left(\boldsymbol{r}-\boldsymbol{r}^{\prime}\right) \rho^{\mathrm{HF}}\left(r_{1} r^{\prime}\right) .
$$


Here $\rho^{\mathrm{HF}}\left(r r^{\prime}\right)$ is the Hartree-Fock approximation to the density matrix and is defined as

$$
\rho\left(r r^{\prime}\right)=\left\langle 0^{N}\left|\psi^{\dagger}(r) \psi\left(r^{\prime}\right)\right| 0^{N}\right\rangle .
$$

In equation (16) $X_{n}^{\mathrm{RPA}}\left(r r^{\prime}\right)$ is the RPA to the transition density matrix defined by

$$
X_{n}\left(r r^{\prime}\right)=\left\langle 0^{N}\left|\psi^{\dagger}(r) \psi\left(r^{\prime}\right)\right| n^{N}\right\rangle
$$

and $V_{0 n}^{\mathrm{RPA}}\left(r r^{\prime}\right)$ is defined by

$$
\begin{aligned}
& \int V_{0 n}^{\mathrm{RPA}(}\left(r r^{\prime}\right) \phi\left(r^{\prime}\right) \mathrm{d} r^{\prime} \\
& \quad=\int V\left(r-r_{1}\right) X_{n}\left(r_{1} r_{1}\right) \mathrm{d} r_{1} \phi(r)-\int V\left(\boldsymbol{r}-\boldsymbol{r}_{1}\right) X_{n}\left(r r_{1}\right) \phi\left(r_{1}\right) \mathrm{d} r_{1} .
\end{aligned}
$$

Equation (16) is the final result of the present work. It is a combination of the ground-state first-order Bethe-Goldstone pair equation (equation (9) of Meneses and Csanak (1975)) and of the RPA equation (see e.g. equation (16a) and (16b) of Csanak (1974)). It contains the RPA transition amplitudes $X_{n}^{\mathrm{RPA}}\left(r_{1} r^{\prime}\right)$ and the ground-state pair amplitude $\chi_{l}^{\mathrm{BG1}}\left(r_{1} r_{2}\right)$, and hence presupposes that these two problems have already been solved. Equation (16) can be readily solved by projecting $\chi_{l}^{n}\left(r_{1} r_{2}\right)$ on to a discrete basis. If equation (16) is solved for a large number of distinct pairs, it can be combined with the appropriate ground-state correlation functions $\chi_{l}\left(r_{1} r_{2}\right)$ to give the two-electron transition density through the relation

$$
\begin{aligned}
\sum_{l} \chi_{l}^{*}\left(r_{1} r_{2}\right) \chi_{l}^{n}\left(r_{1} r_{2}\right) \\
=\sum_{l}\left\langle 0^{N}\left|\psi^{\dagger}\left(r_{2}\right) \psi^{\dagger}\left(r_{1}\right)\right| l^{N-2}\right\rangle\left\langle l^{N-2}\left|\psi\left(r_{1}^{\prime}\right) \psi\left(r_{2}^{\prime}\right)\right| n^{N}\right\rangle \\
=\left\langle 0^{N}\left|\psi^{\dagger}\left(r_{2}\right) \psi^{\dagger}\left(r_{1}\right) \psi\left(r_{1}^{\prime}\right) \psi\left(r_{2}\right)\right| n^{N}\right\rangle \\
=\rho_{n}\left(r_{1} r_{2} r_{1}^{\prime} r_{2}^{\prime}\right)
\end{aligned}
$$

assuming that the number of pairs, i.e. l's, can be considered complete. From $\rho_{n}\left(r_{1} r_{2} r_{1}^{\prime} r_{2}^{\prime}\right)$, with a single contraction and integration one can obtain the one-particle transition density matrix, using the identity

$$
\begin{aligned}
\int \mathrm{d} r_{1}\left\langle 0^{N}\left|\psi^{\dagger}\left(r_{2}\right) \psi^{\dagger}\left(r_{1}\right) \psi\left(r_{1}\right) \psi\left(r_{2}^{\prime}\right)\right| n^{N}\right\rangle \\
=\left\langle 0^{N}\left|\psi^{\dagger}\left(r_{2}\right) N \psi\left(r_{2}^{\prime}\right)\right| n^{N}\right\rangle \\
=(N-1)\left\langle 0^{N}\left|\psi^{\dagger}\left(r_{2}\right) \psi\left(r_{2}^{\prime}\right)\right| n^{N}\right\rangle .
\end{aligned}
$$

Consequently we have

$$
X_{n}^{\mathrm{BG} 1}\left(r_{2} r_{2}^{\prime}\right)=\frac{1}{N-1} \int \rho_{n}^{\mathrm{BG} 1}\left(r_{1} r_{2} r_{1}^{\prime} r_{2}^{\prime}\right) \mathrm{d} r_{1} .
$$

\section{Acknowledgments}

The financial support of the Conselho Nacional de Pesquisas (Brasil) and of the National Science Foundation (USA) is gratefully acknowledged. The authors also 
want to acknowledge the interest and continuing support of Professor Sergio Porto in this research.

\section{References}

Altick P L and Glassgold A E 1964 Phys. Rev. $133632-46$

Amusia M Ya and Cherepkov N A 1975 Case Studies in Atomic Physics vol 5 (Amsterdam: North-Holland) pp $47-179$

Baym G and Kadanoff L P 1961 Phys. Rev. 124 287-99

Bethe H A and Goldstone J 1957 Proc. R. Soc. A 238 551-67

Csanak Gy 1974 J. Phys. B: Atom. Molec. Phys. 7 1289-97

Csanak Gy, Taylor H S and Yarris R 1971 Advances in Atomic and Molecular Physics vol 7 (New York: Academic Press) pp 287-361

Dalgarno A 1966 Perturbation Theory and its Applications in Quantum Mechanics ed C H Wilcox (New York: Wiley) pp 145-83

Dalgarno A and Victor G A 1966 Proc. R. Soc. A $291291-5$

Dunning T H and McKoy V 1967 J. Chem. Phys. 47 1735-47

Freed K F 1968 Phys. Rev. 173 1-24

Gell-Mann M and Low F E 1951 Phys. Rev. 84 350-4

Kato T, Kobayashi T and Namiki M 1960 Progr. Theor. Phys., Suppl. 15 3-60

Kelly H P 1963 Phys. Rev. $131684-99$

- 1964 Phys. Rev. 136 B896-912

Lane A M 1964 Nuclear Theory (New York: Benjamin) chaps 8, 9; pp 94-115

Löwdin P O 1959 Adv. Chem. Phys. 2 207-322

McCurdy C W Jr, Rescigno T M, Yeager D L and McKoy V 1977 Modern Theoretical Chemistry vol 3 (New York: Plenum)

McKoy V and Sinanoğlu O 1964 J. Chem. Phys. 41 2677-88

McLachlan A D and Ball M A 1964 Rev. Mod. Phys. 36 844-55

Martin P C and Schwinger J 1959 Phys. Rev. 115 1342-73

Meneses G D and Csanak Gy 1975 J. Phys. B: Atom. Molec. Phys. 8 L395-9

Nesbet R K 1969 Adv. Chem. Phys. 14 1-34

Parr R G 1963 Quantum Theory of Molecular Electronic Structure (Reading, Mass: Benjamin)

Rowe D J 1968 Rev. Mod. Phys. 153-66

Schaefer H F 1972 The Electronic Structure of Atoms and Molecules (Reading, Mass: Addison-Wesley)

Sinanoğlu O 1961 Proc. R. Soc. A $260379-92$

-1962a J. Chem. Phys. 36 706-17

-1962b J. Chem. Phys. 36 3198-208

Sinanoğlu O and Brueckner K A 1970 Three Approaches to Electron Correlation in Atoms (New Haven and London: Yale University Press)

Sinanoǧlu O and Tuan D F 1963 J. Chem. Phys. 38 1740-48

Tuan D F and Sinanoğlu O 1964 J. Chem. Phys. 41 2677-88

Wendin G 1976 Photoionization and Other Probes of Many-Electron Interactions ed F J Wuilleumier, NATO Advanced Study Institutes, Series B (New York: Plenum)

Yoshizumi H 1959 Adv. Chem. Phys. 2 323-65 